\title{
Exosomes and Exosomal circRNAs: The Rising Stars in the Progression, Diagnosis and Prognosis of Gastric Cancer
}

Ling $\mathrm{Lu}^{\mathrm{l}, *}$

Shikun Fang ${ }^{2, *}$

Yue Zhang $\mathbb{1 D}^{2}$

Longtao Jin'

Wenrong $\mathrm{Xu}^{2}$

Zhaofeng Liang $\mathbb{D}^{2}$

'Child Healthcare Department, The Fourth Affiliated Hospital of Jiangsu University, Zhenjiang, Jiangsu, 2I200I, People's Republic of China; ${ }^{2}$ jiangsu Key Laboratory of Medical Science and Laboratory Medicine, School of Medicine, Jiangsu University, Zhenjiang, Jiangsu, 2/2013, People's Republic of China

*These authors contributed equally to this work
Correspondence: Zhaofeng Liang Jiangsu Key Laboratory of Medical Science and Laboratory Medicine, School of Medicine, Jiangsu University, 30I Xuefu Road, Zhenjiang, Jiangsu, 212013, People's Republic of China

Email liangzhaofeng@ujs.edu.cn

\begin{abstract}
Gastric cancer (GC) is a common malignant tumor affecting human health, with occult onset and poor prognosis. Exosomes are extracellular vesicles secreted by almost all cells, which can reflect the state of source cells or tissues. It is reported that exosomes are involved in almost all processes of GC. Exosomes provided a window to understand changes in cell or tissue states by carrying active components such as circular RNAs (circRNAs). CircRNAs are a naturally occurring class of endogenous noncoding RNAs and abnormal expression during the occurrence and development of GC. Exosomal circRNAs are those circRNAs stably existing in exosomes and having high clinical values as novel potential diagnosis and prognosis biomarkers of GC, which have the characteristics of abnormal expression, tissue specificity and development stage specificity. Herein, we briefly summarize the functions and roles and the current research progress of exosomes and exosomal circRNAs in GC with a focus on the potential application for GC progression, diagnosis and prognosis. We also prospected the clinical application of exosomes and exosomal circRNAs in the future.
\end{abstract}

Keywords: gastric cancer, exosomes, circRNAs, function

\section{Introduction}

Gastric cancer (GC) is the fourth most common cancer in the world, and the third leading cause of cancer death in the world. There are about 1.089 million new GC cases and 768,000 deaths every year. ${ }^{1}$ Although great progress has been made in therapeutic methods such as surgery combined with chemotherapy, radiotherapy and immunotherapy, the prognosis of patients with advanced GC is still poor and lack of indicators for early diagnosis. GC is often asymptomatic in the early stage, which may delay early diagnosis and miss opportunity of effective treatment. Therefore, looking for new non-invasive biomarkers with high sensitivity and specificity for early GC screening is very important to improve the quality of life and prognosis of GC patients. In recent years, the role of exosomes in the diagnosis, progression, treatment and prognosis of GC has attracted more and more attention.

Exosomes, extracellular vesicles of $30 \sim 150 \mathrm{~nm}$ in diameter, are present in almost all body fluids, such as blood and urine. ${ }^{2,3}$ Almost all human cells can secrete exosomes, which are released into the extracellular after fusion with the plasma membrane. ${ }^{4}$ Exosomes are enriched in selected many active components, such as protein, lipid, nucleic acid, glycoconjugates, metabolites and so on, and 
play an important role in many physiological and pathological processes. ${ }^{4,5}$ In recent years, more and more attention has been attracted to miRNA, IncRNA, circRNA and other exosomal RNAs in tumor tumorigenesis, development, diagnosis, and prognosis. ${ }^{6}$ These exosomal RNAs can be absorbed by the neighboring cells or distant cells, and then regulate some physiological or pathological processes of the receptor cells. The discovery of the function of exosomes carrying active components in genetic exchange between cells has brought more and more attention to exosomes. Emerging evidence has shown that exosomes and their cargos have been applied as novel biomarkers for $\mathrm{GC}$ diagnosis and prognosis.

CircRNAs are a naturally occurring class of endogenous noncoding RNAs, which play an important role in many physiological and pathological processes. ${ }^{7,8}$ CircRNAs are abundant, stable, tissue specificity, and it has been found that thousands of circRNAs are differentially expressed between tumor tissues and normal tissues. ${ }^{8,9}$ Accumulating studies have suggested that circRNAs function as vital players in multiple human diseases, including GC. In addition, compared with linear RNAs, circRNAs have more stable properties and longer half-lives, which determines that circRNAs are more suitable as biomarkers than linear RNAs. ${ }^{10}$ Although studies have demonstrated that circRNAs are enriched and stable in exosomes, the biological function of exosomal circRNAs in the occurrence, development, diagnosis and prognosis of GC is still unclear. ${ }^{11-13}$

In this review, we highlight and discuss about the relationship between exosomes, exosomal circRNAs and GC, with a special focus on the roles, mechanisms of actions, and potential clinical application values as biomarkers and therapeutic targets in GC. Our review provides insight into the role exosomes and exosomal circRNAs in the occurrence, development and prognosis of GC, which may provide a new entry point for discovering new diagnostic and prognosis markers and therapeutic strategies.

\section{Role of Exosomes in the Progress, Diagnosis, and Prognosis of GC}

Exosomes' diverse constituents include nucleic acids, proteins, lipids, amino acids, and metabolites, which can reflect the state of source cells. The potential biological functions of exosomes in medicine have been studied and demonstrated. Exosomes have been reported to be involved in each process of various cancers including GC, such as angiogenesis, metastasis, EMT, immune escape, chemotherapy resistance. ${ }^{6,14,15}$ Exosomes have attracted the increasingly interest in medicine, especially as biomarkers for cancer clinical diagnosis.

\section{Exosomes Mediated the Occurrence and Development of GC}

Exosomes have shown a great potential for acting as effective biomarkers in the occurrence and development of GC, which provided new ideas about how exosomes used to diagnosis and treat GC efficiently.

Integrin $\alpha 6$ and $\alpha \mathrm{X}$ protein play a key role in the specific uptake of exosomes from gastric epithelial cells. On this basis, exosomal GKN1 protein inhibited the carcinogenesis of GC by downregulating HRas/Raf/MEK/ERK pathway. ${ }^{16}$ $\mathrm{Qu}$ et al described that SGC-7901 cells-derived exosomes promoted GC cell proliferation through PI3K/Akt and MAPK/ERK activation for the first time in $2009 .{ }^{17}$ In 2012, preliminary research of our research group found that the GC exosomes trigger the differentiation of umbilical cord MSCs into cancer-related fibroblasts mediated by TGF$\beta /$ Smad pathway. ${ }^{18}$ Our preliminary study demonstrated that exosomal TRIM3 overexpression reduced the occurrence and metastasis of GC through the regulation of stem cells and EMT factors. ${ }^{19}$ LncHEIH encapsulated in exosomes was released by GC cells and then absorbed by normal gastric cells. ${ }^{20}$ The uptake of lncHEIH leads to the up regulation of EZH2 and promotes the malignant transformation of normal gastric cells. Shi et al confirmed that cancer-associated fibroblast-derived exosomes played important roles in promoting the proliferation, migration and invasion of GC cells by delivering circ_0088300 to GC cells. ${ }^{21}$ It is reported that GC-cells-derived exosomes promoted the growth and metastasis of GC by transporting Forkhead box protein M1, suggesting that exosomal Forkhead box protein M1 may be used as a potential biomarker for the diagnosis and treatment of GC. ${ }^{22}$ Chang et al reported that exosomes are the potential nanocarriers for regulating the expression of miR-1228 and exosomes derived from miR-1228 overexpressing cells inhibited the growth of GC cells. ${ }^{23}$ These studies have shown that exosomes play an important role in the occurrence and development of $\mathrm{GC}$ and can be used as potential diagnostic markers of GC.

\section{Exosomes Played an Important Role in Invasion and Metastasis of GC}

Exosomes could mediate GC invasion and metastasis to local or distant tissues and organs by changing the cancer 
microenvironment and producing long-distance effects. ${ }^{14,24}$ The interaction between cancer cells, peritoneal mesothelial cells (PMCs) and lymph node cells can accelerate the invasion and metastasis of $\mathrm{GC}$, in which exosomes play an important role.

Our group found that GC-cells-derived exosomes activate $\mathrm{NF}-\kappa \mathrm{B}$ pathway in macrophages to promote cancer progression. $^{25}$ GC-cells-derived exosomes could induce infiltration of PMCs, and the infiltration of PMCs could promote the invasion of tumor to subserosal in turn. ${ }^{26}$ Data from $\mathrm{Li}$ et al suggested that exosomal miR-21-5p induces mesothelial-to-mesenchymal transition of PMCs and promotes GC peritoneal metastasis by targeting SMAD7. ${ }^{27}$

Lymph node metastasis-GC cells could specifically educate BM-MSCs to promote lymph node metastasis of GC via exosomal Wnt5a-elicited activation of YAP. ${ }^{28}$ Another study has shown that exosomal TGF- $\beta 1$ plays an important role in lymph node metastasis and the ratio of Treg cells in lymph nodes of GC. ${ }^{29}$ The data of Xia indicated that exosomes rich in miR-301a-3p secreted by GC cells under hypoxic TME, which was helpful for HIF$1 \alpha$ accumulation and promotion of malignant behavior and metastasis of GC. ${ }^{30}$

These results indicated that exosomes can promote the invasion and metastasis of GC by participating in intercellular communication and influencing tumor microenvironment. Exosomes and their active molecules may be a promising predictor of GC metastasis and a potential therapeutic target.

\section{Exosomes and Drug Resistance in GC}

Drug resistance is a major clinical problem, which partially leads to poor survival rate of GC patients. ${ }^{31}$ However, drug resistance limits its effectiveness for the treatment of GC. Sun et al demonstrated that cisplatin-resistant GC cells derived exosomes enhanced the chemoresistance of cisplatin-sensitive GC cells through exosomal RPS3 mediated PI3K/Akt/cofilin-1 pathway. ${ }^{31}$ Therefore, targeting exosomal RPS3 in cisplatin resistant GC cells may be a promising strategy to overcome cisplatin resistance. The findings of Zheng et al suggested that exosomal transfer of cancer-associated macrophages derived miR-21 mediates cisplatin resistance in $\mathrm{GC}^{32} \mathrm{C}$-Met is overexpressed in $\mathrm{GC}$, which leads to poor prognosis of GC. It is reported that exosome delivered c-Met siRNA can inhibit the growth, invasion and metastasis of GC cells and promote apoptosis, and then reverse the resistance to cisplatin in
GC. ${ }^{33}$ Study has shown that cancer-associated fibroblasts secrete exosomal miR-522 to inhibit ferroptosis in GC cells through targeting ALOX15 and blocking lipid-ROS accumulation, which reveals a new mechanism of acquired chemo-resistance in GC. ${ }^{34}$ MiR-501 was overexpressed in doxorubicin-resistant GC cells-secreted exosomes, and the further results showed that exosomal transfer of miR-501 can confer doxorubicin-resistance in GC. ${ }^{35}$

Exosomes and their active components played an important role in drug resistance of GC. Exploring the mechanism of exosomes and mediated drug resistance is expected to improve the drug resistance of GC patients and improve the treatment efficiency of GC patients.

\section{Exosomes and Early Diagnosis of GC}

The age standardized five-year survival rate of GC is reported $20-40 \%$ due to late diagnosis, poor prognosis and low efficiency of therapies. ${ }^{9,36}$ Therefore, it is particularly important to investigate early and effective diagnostic markers for GC. Exosomes played important roles in human health and diseases, including GC, owing to the ability to carry various bioactive molecules, data transmission and cell reprogramming. ${ }^{10,36}$ Exosomes provided a window into altered cellular or tissue states by carrying active components and detection of these components in biological fluid may provide early and effective diagnosis for GC patients. ${ }^{4,37}$

Kahroba et al summarized the main roles of key exosomal substances in the exosome-mediated signal transduction of GC cells and the important significance of exosomes in body fluid in the early diagnosis of GC. ${ }^{36}$ Guo et al demonstrated that exosomal lncRNAGC1 exhibited has higher specificity and sensitivity for the early detection of GC compared with CEA, CA72-4, and CA19-9 $(\mathrm{AUC}=0.9033)$, especially for GC patients with negative standard biomarkers. ${ }^{38}$ The accuracy of exosomal lncUEGC1 in the early diagnosis of GC was evaluated and the AUC value of exosomal lncUEGC1 was 0.8760 and 0.8406 , respectively, in discriminating early GC patients from healthy people and precancerous chronic atrophic gastritis patients, which was higher than the diagnostic accuracy of CEA. ${ }^{39}$ Serum exosomal miR-92b-3p, miR-146b-5p, miR-9-5p, and miR-let-7g-5p have been proven to be potential non-invasive biomarkers for early diagnosis of GC. ${ }^{40}$

The potential functions of exosomes as novel early diagnostic biomarkers for GC are studied extensively. It is believed that exosomes may act as non-invasive 
biomarkers, which can provide insights into the early diagnosis for GC.

\section{Exosomes and Prognosis of GC}

Early symptoms of GC are not obvious, generally diagnosed as advanced, leading to poor prognosis of GC. Therefore, it is very important to find the markers that can judge the prognosis of $\mathrm{GC}$ in time to improve the quality of life of patients with GC. Exosomes and their cargo also play an important role in the prognosis of GC.

It is reported that exosomal HOTTIP was an independent prognostic factor of $\mathrm{GC}(\mathrm{P}=0.027)$, which revealed exosomal HOTTIP may be a potential GC prognosis biomarker. $^{41}$ Zheng et al demonstrated that exosomal miR-590-5p was related to clinical stage of GC ( $\mathrm{P}=$ $0.008)$ and overall survival rate $(\mathrm{P}<0.001){ }^{42}$ The results of Kumata et al indicated that exosomal miR-23b was an independent prognostic factor for overall survival rates and disease-free survival rates of GC, which has potential to be a predictive biomarker for the prognosis of GC. ${ }^{43}$

\section{Exosomal circRNAs Play Important Roles in the Progression, Diagnosis and Prognosis of GC}

By delivering circRNAs, miRNAs, IncRNAs, proteins and other active ingredients, exosomes play a key role in mediating signaling transduction between neighboring or distant cells and tissues. ${ }^{5,6}$ A large number of studies have proved that circRNAs are enriched and stable in exosomes from various sources. Exosomal circRNAs have important values in the progression, diagnosis and prognosis of GC and other cancers. It has been demonstrated that exosomal circRNAs can distinguish patients with cancer from healthy people, and exosomal circRNAs can become very promising biomarkers for liquid biopsy., ${ }^{9,44,45}$ Exosomal circRNAs were associated with advanced TNM stage, metastases, invasion, poor prognosis and worse survival of GC, which can be stable biomarkers for GC diagnosis and prognosis. ${ }^{9,46}$ Multiple studies have demonstrated that numerous cancer-derived or cancerassociated exosomal circRNAs could be reliable specific biomarkers of GC. . $^{67,48}$

\section{Exosomal circRNAs and Occurrence and Development of GC}

A group of exosomal circRNAs are abnormal expression in GC cells and tissues, which may play important roles in the occurrence and development of GC. CircRNAs are not easy to be degraded by exonucleases and ribonucleases, and so they have greater stability and longer half-life in body fluid. Additionally, the expression of exosomal circRNAs often provided relatively high sensitivity and specificity in different stages of GC. ${ }^{47,49}$

These findings of Xie demonstrated that exosomal circSHKBP1 regulate the miR-582-3p/HUR/VEGF pathway to promote $\mathrm{GC}$ progression and circSHKBP1 may be a promising circulating biomarker for GC diagnosis. ${ }^{9}$ Shi et al found that exosomal circ 0088300 derived from cancer-associated fibroblast cells acts as a sponge of miR1305 to promote GC cells proliferation, migration and invasion. ${ }^{21}$ Exosomal circ0044366 was highly expressed in GC, which could significantly inhibit the proliferation, migration and tube formation of HUVEC. Further through regulation of VEGF, exosomal circ0044366 played a responsibility in the occurrence and development of GC. ${ }^{50}$ CircNEK9 was upregulated in GC tissues and cells. Yu et al reported that exosome-triggered transfer of circNEK9 accelerated the proliferation, migration and invasion of GC cells by targeting miR-409-3p. ${ }^{11}$ CircNHSL1 was highly expressed in GC tissues, GC cells and GC cells derived exosomes. Study disclosed that knockdown of circNHSL1 represses the migration and invasion in GC cells and inhibits tumor growth, implying a potential diagnostic marker for the occurrence and development of GC. ${ }^{51}$ CircNRIP1 was highly expressed in GC, which might play a role in promoting GC. It was proven that circNRIP1 can be transmitted by exosomal communication between GC cells and exosomal circNRIP1 promotes progression and metastasis of GC via the miR-149-5p/AKT1/mTOR pathway. ${ }^{52}$ GC-derived exosomes can induce the expression of circ_0004303 to promote the migration and homing of MSCs in adjacent tissues regulates the biological function of MSCs, so as to promote the progress of $\mathrm{GC}^{53} \mathrm{CircITCH}$ was downregulated in GC cell lines, GC tissues and serum-derived exosomes. CircITCH suppressed the metastasis of GC by acting as the sponge of miR-199a-5p and increasing Klotho expression, which serves as a potential biomarker for the occurrence and development of $\mathrm{GC} .{ }^{54} \mathrm{Lu}$ et al indicated that circRanGAP1 was upregulated in GC tissues and serum-derived exosomes. CircRanGAP1 mediated miR-877-3p/VEGFA axis to facilitate GC invasion and metastasis. ${ }^{46}$ Jiang et al found that CDR1as plays an important suppressive role in GC metastasis through miR-876-5p/GNG7 axis. $^{55}$ 
CircRNAs are highly conserved and specific in tissue, developmental stage and tumor type, which can play an important role in the occurrence and development of GC. Exosomes protect the degradation of circRNAs and facilitate the transmission of GC-related circRNAs. Therefore, exosomal circRNAs are crucial in the occurrence and development of GC. These results identified exosomal circRNAs can be used as potential biomarkers for the occurrence and development of GC.

\section{Role of Exosomal circRNAs in the GC Diagnosis}

Exosomes could provide the basis for liquid biopsies to detect cancer and monitor treatment response. ${ }^{56}$ Normal and cancer cells shed exosomes packed with proteins, lipid finger, nucleic acid (miRNA, IncRNA, circRNA and other RNAs), glycoconjugates and other molecules. Researchers have been investigating whether circRNAs in exosomes could serve as biomarkers for diagnosing GC. Exosomal circRNAs have significant heterogeneity, which provides a possibility for the diagnosis, staging and treatment of tumors.

Circ0065149 is one of the dysregulated circRNAs in GC carcinogenesis, which may be a diagnosis biomarker for early GC. Circ0065149 expression levels in GC tissues were significantly associated with tumor diameter $(\mathrm{P}=$ $0.034)$ and perineural invasion $(\mathrm{P}=0.037) .{ }^{57}$ More importantly, as a screening biomarker for early GC, exosomal circ0065149 in plasma has higher sensitivity and specificity than CEA and CA19-9. ${ }^{57}$ Circ0000419 exists in exosomes and remains stable, which makes it possible for circ0000419 to become a diagnostic marker for GC. Circ0000419 were significantly correlated with GC, the sensitivity and specificity of circ0000419 were 0.682 and $0.884 .^{58}$ These studies suggested that there was a great hope and broad clinical application prospects in the diagnosing GC through exosomal circRNAs (Table 1).

\section{Exosomal circRNAs and Drug Resistance and Prognosis of GC}

Exosomal circRNAs have emerged recently as key regulators in human cancers including GC. It is believed that exosomal circRNAs may act as non-invasive biomarkers, which can provide the prognosis and drug resistance information of GC patients, so as to improve the quality of life and life cycle of GC patients.

Increased expression of exosomal circSHKBP1 was related to advanced TNM stage, poor survival, and the level of exosomal circSHKBP1 significantly decreased after gastrectomy. ${ }^{9}$ These results suggested that exosomal circSHKBP1 is a promising biomarker for GC prognosis. The expression of plasma exosomal circ0065149 levels were significantly decreased in the early GC patients. ${ }^{57}$ Circ0065149 levels are closely related to the prognosis and survival of patients with GC. Exosomal circ0065149 can be used as an early predictor of the prognosis of GC.

CircPVT1 was upregulated in cisplatin-resistant GC serums and cell exosomes. Exosomal circPVT1 boosted cisplatin resistance of GC cells via regulating autophagy, invasion and apoptosis through miR-30a-5p/YAP1 axis. ${ }^{59}$ The expression of circ0000260 was increased in cancer tissues and serum-derived exosomes of GC patients and circ0000260 knockdown lessened cisplatin chemoresistance. ${ }^{60}$ The expression of circ0032821 was promoted in oxaliplatinresistant GC cells secreted exosomes. Exosomal circ0032821 facilitated oxaliplatin resistance of GC cells via targeting the miR-515-5p/SOX9 pathway. ${ }^{12}$

Exosomal circRNAs were associated with advanced TNM stage, drug resistance, poor prognosis, worse survival of GC, which can be stable biomarkers for GC resistance and prognosis. After further research and improvement, exosomes-derived circRNAs are expected to be effective markers for improving drug resistance and prognosis of GC in clinical application (Table 1).

\section{Summary and the Future Challenges}

In the past few years, our understanding of exosomes and exosomal circRNAs has advanced rapidly. Based on the above research, we found that exosomes and exosomal circRNAs play critical roles in the progression, diagnosis, drug resistance and prognosis of GC (Figure 1 and Table 1). There is great hope that exosomes and exosomes derived circRNAs may be used in the diagnosis, prognosis and treatment of GC. However, there are still many unknowns that need to be explored, and many improvements and challenges in the future research and clinical application.

Firstly, the mechanism of exosomes secreting and encapsulating circRNAs, which play an important role in the progression, drug resistance and prognosis of $\mathrm{GC}$, is not very clear. Secondly, the mechanism of cyclization, degradation and beneficiation of GC-related exosomal circRNAs is still unclear. Besides, there is a lack of standardized methods for the extraction/detection of exosomes 
Table I Overview of the Identified GC-Associated Exosomal circRNAs

\begin{tabular}{|c|c|c|c|c|c|}
\hline CircRNA & Expression & Putative Function & Target & Type of Biomarker & References \\
\hline CircSHKBPI & Upregulated & $\begin{array}{l}\text { Promoted proliferation, migration, invasion } \\
\text { and angiogenesis }\end{array}$ & $\begin{array}{l}\text { MiR-582-3p/ } \\
\text { HUR/VEGF }\end{array}$ & $\begin{array}{l}\text { Occurrence, development } \\
\text { and prognosis }\end{array}$ & [9] \\
\hline CircNEK9 & Upregulated & $\begin{array}{l}\text { Accelerated the proliferation, migration and } \\
\text { invasion }\end{array}$ & $\begin{array}{l}\text { MiR-409-3p/ } \\
\text { MAP7 }\end{array}$ & $\begin{array}{l}\text { Occurrence and } \\
\text { development }\end{array}$ & {$[11]$} \\
\hline Circ0088300 & Upregulated & $\begin{array}{l}\text { Promoted proliferation, migration and } \\
\text { invasion }\end{array}$ & $\begin{array}{l}\text { MiR-I305/ } \\
\text { JAKI /STATI }\end{array}$ & $\begin{array}{l}\text { Occurrence, development } \\
\text { and prognosis }\end{array}$ & {$[21]$} \\
\hline Circ0044366 & Upregulated & Promoted angiogenesis & MiR-29a/VEGF & $\begin{array}{l}\text { Development and } \\
\text { treatment }\end{array}$ & [49] \\
\hline CircRanGAPI & Upregulated & Facilitate GC invasion and metastasis & $\begin{array}{l}\text { MiR-877-3p/ } \\
\text { VEGFA }\end{array}$ & $\begin{array}{l}\text { Occurrence and } \\
\text { development }\end{array}$ & [46] \\
\hline CircNHSLI & Upregulated & $\begin{array}{l}\text { Promoted migration, invasion and tumor } \\
\text { growth }\end{array}$ & $\begin{array}{l}\text { MiR-I49-5p/ } \\
\text { YWHAZ }\end{array}$ & Progression & {$[50]$} \\
\hline CircNRIPI & $\begin{array}{l}\text { Highly } \\
\text { expressed }\end{array}$ & $\begin{array}{l}\text { Promoted proliferation, migration and } \\
\text { invasion }\end{array}$ & $\begin{array}{l}\text { MiR-I49-5p/ } \\
\text { AKTI/mTOR }\end{array}$ & $\begin{array}{l}\text { Occurrence and } \\
\text { development }\end{array}$ & {$[51]$} \\
\hline Circ0004303 & Upregulated & Promote GC growth & $\begin{array}{l}\text { MiR-I48a-3P/ } \\
\text { ALCA }\end{array}$ & $\begin{array}{l}\text { Occurrence and } \\
\text { development }\end{array}$ & [53] \\
\hline CirCDRlas & Upregulated & Suppressed the metastasis of GC & $\begin{array}{l}\text { miR-876-5p/ } \\
\text { GNG7 }\end{array}$ & $\begin{array}{l}\text { Occurrence and } \\
\text { development }\end{array}$ & {$[55]$} \\
\hline CirclTCH & Downregulated & Suppressed the metastasis of GC & $\begin{array}{l}\text { miR- } 199 a-5 p / \\
\text { Klotho }\end{array}$ & $\begin{array}{l}\text { Occurrence and } \\
\text { development }\end{array}$ & {$[54]$} \\
\hline Circ0065I49 & Downregulated & $\begin{array}{l}\text { Significantly associated with tumor diameter } \\
\text { and perineural invasion }\end{array}$ & & Diagnosis and prognosis & {$[57]$} \\
\hline Circ00004I9 & Downregulated & $\begin{array}{l}\text { Associated with differentiation, tumor stage, } \\
\text { metastasis and invasion }\end{array}$ & & Diagnosis and prognosis & {$[58]$} \\
\hline CircPVTI & Upregulated & Boosted cisplatin resistance of GC & $\begin{array}{l}\text { MiR-30a-5p/ } \\
\text { YAPI }\end{array}$ & Drug resistant & [59] \\
\hline Circ0000260 & Upregulated & Boosted cisplatin resistance of GC & & Drug resistant & {$[60]$} \\
\hline Circ003282I & $\begin{array}{l}\text { Highly } \\
\text { expressed }\end{array}$ & Facilitated oxaliplatin resistance of GC & $\begin{array}{l}\operatorname{miR}-515-5 p / \\
\text { SOX9 }\end{array}$ & Drug resistant & {$[12]$} \\
\hline
\end{tabular}

and exosome derived circRNAs in clinical application. The clinical application of exosomes and exosomal circRNAs in the progression, diagnosis, drug resistance and prognosis of GC needs more detailed and complete data. Systematic evaluation of the cost, accuracy, repeatability, specificity and sensitivity of exosomes and exosomal circRNAs in large GC samples needs to be further strengthened and improved. In addition, it is not very clear whether exosomes and exosomal circRNAs can affect GC microenvironment cells and affect the progression, drug resistance and prognosis of GC. These challenges and deficiencies provide direction for future research, technology development and clinical application. We believe that with the progress of research, the update of biomedical technology, these challenges and problems will eventually be solved, so as to promote the clinical application of exosomes and exosomal circRNAs.

Based on the above research, we also speculate the potential future directions of exosomes and exosomal circRNAs in the field of medicine. Firstly, as new clinical biomarkers of GC, exosomes and exosomal circRNAs will become the cornerstone of new clinical laboratory analysis methods. By detecting exosomes and exosomal circRNAs, clinicians can diagnose early $\mathrm{GC}$, monitor the metastasis and recurrence of 


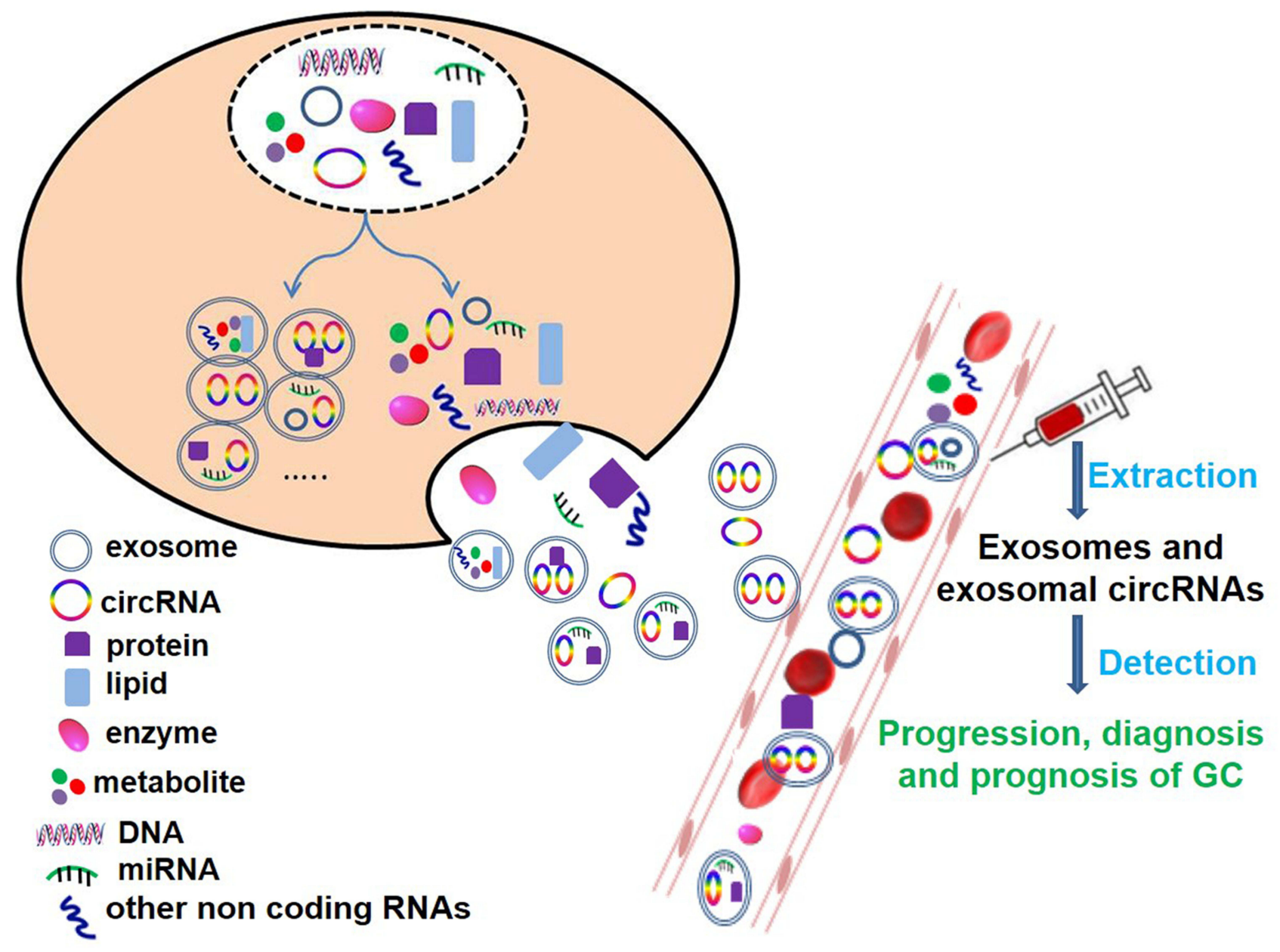

Figure I CircRNAs and exosomal circRNAs play a very important role in the diagnosis, progression and prognosis of GC.

GC, observe the drug resistance of GC, and predict the prognosis and survival time. Secondly, as potential therapeutic targets of GC, exosomes and exosome-derived circRNAs can help clinical doctors prolong the life of patients with advanced GC and improve the quality of life of GC patients. We look forward to the day of application of exosomes and exosomal circRNAs in the diagnosis, prognosis and treatment of GC.

\section{Acknowledgments}

This work was supported by project of social development in Zhenjiang (No. SH2021045, FZ2019038), the Foundation for Excellent Young Teachers of Jiangsu University, and Clinical Medical Science and Technology Development Foundation of Jiangsu University (No. JLY2021013).

\section{Disclosure}

The authors have no conflicts of interest in this work.

\section{References}

1. Arnold M, Abnet CC, Neale RE, et al. Global burden of 5 major types of gastrointestinal cancer. Gastroenterology. 2020;159(1):335-349e315.

2. Nam GH, Choi Y, Kim GB, Kim S, Kim SA, Kim IS. Emerging prospects of exosomes for cancer treatment: from conventional therapy to immunotherapy. Adv Mater. 2020;32(51):e2002440.

3. Hoshino A, Kim HS, Bojmar L, et al. Extracellular vesicle and particle biomarkers define multiple human cancers. Cell. 2020;182(4):1044$1061 \mathrm{e} 1018$.

4. Kalluri R, LeBleu VS. The biology, function, and biomedical applications of exosomes. Science. 2020;367:6478.

5. Pegtel DM, Gould SJ. Exosomes. Annu Rev Biochem. 2019;88: 487-514.

6. Zhang H, Zhu L, Bai M, et al. Exosomal circRNA derived from gastric tumor promotes white adipose browning by targeting the miR-133/ PRDM16 pathway. Int J Cancer. 2019;144(10):2501-2515.

7. Chen LL. The expanding regulatory mechanisms and cellular functions of circular RNAs. Nat Rev Mol Cell Biol. 2020;21(8):475-490.

8. Kristensen LS, Andersen MS, Stagsted LVW, Ebbesen KK, Hansen TB, Kjems J. The biogenesis, biology and characterization of circular RNAs. Nat Rev Genet. 2019;20(11):675-691.

9. Xie M, Yu T, Jing X, et al. Exosomal circSHKBP1 promotes gastric cancer progression via regulating the miR-582-3p/HUR/VEGF axis and suppressing HSP90 degradation. Mol Cancer. 2020;19(1):112. 
10. Hu F, Liu J, Liu H, et al. Role of exosomal non-coding RNAs in gastric cancer: biological functions and potential clinical applications. Front Oncol. 2021;11:700168.

11. Yu L, Xie J, Liu X, Yu Y, Wang S. Plasma exosomal CircNEK9 accelerates the progression of gastric cancer via miR-409-3p/MAP7 axis. Dig Dis Sci. 2021. doi:10.1007/s10620-020-06816-z

12. Zhong Y, Wang D, Ding Y, Tian G, Jiang B. Circular RNA circ_0032821 contributes to oxaliplatin (OXA) resistance of gastric cancer cells by regulating SOX9 via miR-515-5p. Biotechnol Lett. 2021;43(2):339-351.

13. O'Brien K, Breyne K, Ughetto S, Laurent LC, Breakefield XO. RNA delivery by extracellular vesicles in mammalian cells and its applications. Nat Rev Mol Cell Biol. 2020;21(10):585-606.

14. Wu H, Fu M, Liu J, et al. The role and application of small extracellular vesicles in gastric cancer. Mol Cancer. 2021;20(1):71.

15. Zhang X, Shi H, Yuan X, Jiang P, Qian H, Xu W. Tumor-derived exosomes induce $\mathrm{N} 2$ polarization of neutrophils to promote gastric cancer cell migration. Mol Cancer. 2018;17(1):146.

16. Yoon JH, Ashktorab H, Smoot DT, Nam SW, Hur H, Park WS. Uptake and tumor-suppressive pathways of exosome-associated GKN1 protein in gastric epithelial cells. Gastric Cancer. 2020;23 (5):848-862.

17. Qu JL, Qu XJ, Zhao MF, et al. Gastric cancer exosomes promote tumour cell proliferation through PI3K/Akt and MAPK/ERK activation. Dig Liver Dis. 2009;41(12):875-880.

18. Gu J, Qian H, Shen L, et al. Gastric cancer exosomes trigger differentiation of umbilical cord derived mesenchymal stem cells to carcinoma-associated fibroblasts through TGF-beta/Smad pathway. PLoS One. 2012;7(12):e52465.

19. Fu H, Yang H, Zhang X, et al. Exosomal TRIM3 is a novel marker and therapy target for gastric cancer. J Exp Clin Cancer Res. 2018;37(1):162.

20. Lu Y, Hou K, Li M, Wu X, Yuan S. Exosome-delivered LncHEIH promotes gastric cancer progression by upregulating EZH2 and stimulating methylation of the GSDME promoter. Front Cell Dev Biol. 2020;8:571297.

21. Shi H, Huang S, Qin M, et al. Exosomal circ_0088300 derived from cancer-associated fibroblasts acts as a miR-1305 sponge and promotes gastric carcinoma cell tumorigenesis. Front Cell Dev Biol. 2021;9:676319.

22. Zhang Y, Chen L, Ye X, et al. Expression and mechanism of exosome-mediated A FOXM1 related long noncoding RNA in gastric cancer. J Nanobiotechnol. 2021;19(1):133.

23. Chang L, Gao H, Wang L, et al. Exosomes derived from miR-1228 overexpressing bone marrow-mesenchymal stem cells promote growth of gastric cancer cells. Aging. 2021;13(8):11808-11821.

24. Gao J, Li S, Xu Q, et al. Exosomes promote pre-metastatic niche formation in gastric cancer. Front Oncol. 2021;11:652378.

25. Wu L, Zhang $\mathrm{X}$, Zhang $\mathrm{B}$, et al. Exosomes derived from gastric cancer cells activate NF-kappaB pathway in macrophages to promote cancer progression. Tumour Biol. 2016;37(9):12169-12180.

26. Tanaka M, Kuriyama S, Itoh G, et al. Mesothelial cells create a novel tissue niche that facilitates gastric cancer invasion. Cancer Res. 2017;77(3):684-695.

27. Li Q, Li B, Li Q, et al. Exosomal miR-21-5p derived from gastric cancer promotes peritoneal metastasis via mesothelial-tomesenchymal transition. Cell Death Dis. 2018;9(9):854.

28. Wang M, Zhao X, Qiu R, et al. Lymph node metastasis-derived gastric cancer cells educate bone marrow-derived mesenchymal stem cells via YAP signaling activation by exosomal Wnt5a. Oncogene. 2021;40(12):2296-2308.

29. Yen EY, Miaw SC, Yu JS, Lai IR. Exosomal TGF-beta1 is correlated with lymphatic metastasis of gastric cancers. Am J Cancer Res. 2017;7(11):2199-2208.

30. Xia X, Wang S, Ni B, et al. Hypoxic gastric cancer-derived exosomes promote progression and metastasis via MiR-301a-3p/PHD3/HIF1alpha positive feedback loop. Oncogene. 2020;39(39):6231-6244.
31. Sun MY, Xu B, Wu QX, et al. Cisplatin-resistant gastric cancer cells promote the chemoresistance of cisplatin-sensitive cells via the exosomal RPS3-mediated PI3K-Akt-Cofilin-1 signaling axis. Front Cell Dev Biol. 2021;9:618899.

32. Zheng P, Chen L, Yuan $\mathrm{X}$, et al. Exosomal transfer of tumor-associated macrophage-derived miR-21 confers cisplatin resistance in gastric cancer cells. J Exp Clin Cancer Res. 2017;36(1):53.

33. Zhang Q, Zhang H, Ning T, et al. Exosome-delivered c-Met siRNA could reverse chemoresistance to cisplatin in gastric cancer. Int J Nanomedicine. 2020;15:2323-2335.

34. Zhang H, Deng T, Liu R, et al. CAF secreted miR-522 suppresses ferroptosis and promotes acquired chemo-resistance in gastric cancer. Mol Cancer. 2020;19(1):43.

35. Liu X, Lu Y, Xu Y, et al. Exosomal transfer of miR-501 confers doxorubicin resistance and tumorigenesis via targeting of BLID in gastric cancer. Cancer Lett. 2019;459:122-134.

36. Kahroba H, Hejazi MS, Samadi N. Exosomes: from carcinogenesis and metastasis to diagnosis and treatment of gastric cancer. Cell Mol Life Sci. 2019;76(9):1747-1758.

37. Wang J, Liu Y, Sun W, Zhang Q, Gu T, Li G. Plasma exosomes as novel biomarker for the early diagnosis of gastric cancer. Cancer Biomark. 2018;21(4):805-812.

38. Guo X, Lv X, Ru Y, et al. Circulating exosomal gastric cancer-associated long noncoding RNA1 as a biomarker for early detection and monitoring progression of gastric cancer: a multiphase study. JAMA Surg. 2020;155(7):572-579.

39. Lin LY, Yang L, Zeng Q, et al. Tumor-originated exosomal lncUEGC1 as a circulating biomarker for early-stage gastric cancer. Mol Cancer. 2018;17(1):84.

40. Tang S, Cheng J, Yao Y, et al. Combination of four serum exosomal MiRNAs as novel diagnostic biomarkers for early-stage gastric cancer. Front Genet. 2020;11:237.

41. Zhao R, Zhang Y, Zhang X, et al. Exosomal long noncoding RNA HOTTIP as potential novel diagnostic and prognostic biomarker test for gastric cancer. Mol Cancer. 2018;17(1):68.

42. Zheng GD, Xu ZY, Hu C, et al. Exosomal miR-590-5p in serum as a biomarker for the diagnosis and prognosis of gastric cancer. Front Mol Biosci. 2021;8:636566.

43. Kumata Y, Iinuma H, Suzuki Y, et al. Exosomeencapsulated microRNA23b as a minimally invasive liquid biomarker for the prediction of recurrence and prognosis of gastric cancer patients in each tumor stage. Oncol Rep. 2018;40(1):319-330.

44. Li S, Li Y, Chen B, et al. exoRBase: a database of circRNA, IncRNA and mRNA in human blood exosomes. Nucleic Acids Res. 2018;46 (D1):D106-D112.

45. Li Y, Zheng Q, Bao C, et al. Circular RNA is enriched and stable in exosomes: a promising biomarker for cancer diagnosis. Cell Res. 2015;25(8):981-984.

46. Lu J, Wang YH, Yoon C, et al. Circular RNA circ-RanGAP1 regulates VEGFA expression by targeting miR-877-3p to facilitate gastric cancer invasion and metastasis. Cancer Lett. 2020;471:38-48.

47. Wang Y, Li Z, Xu S, Guo J. Novel potential tumor biomarkers: circular RNAs and exosomal circular RNAs in gastrointestinal malignancies. J Clin Lab Anal. 2020;34(7):e23359.

48. Li P, Chen H, Chen S, et al. Circular RNA 0000096 affects cell growth and migration in gastric cancer. Br J Cancer. 2017;116(5):626-633.

49. Yan Y, Fu G, Ye Y, Ming L. Exosomes participate in the carcinogenesis and the malignant behavior of gastric cancer. Scand J Gastroenterol. 2017;52(5):499-504.

50. Li S, Li J, Zhang H, et al. Gastric cancer derived exosomes mediate the delivery of circRNA to promote angiogenesis by targeting miR-29a/VEGF axis in endothelial cells. Biochem Biophys Res Commun. 2021;560:37-44.

51. Hui C, Tian L, He X. Circular RNA circNHSL1 contributes to gastric cancer progression through the miR-149-5p/YWHAZ axis. Cancer Manag Res. 2020;12:7117-7130. 
52. Zhang X, Wang S, Wang $\mathrm{H}$, et al. Circular RNA circNRIP1 acts as a microRNA-149-5p sponge to promote gastric cancer progression via the AKT1/mTOR pathway. Mol Cancer. 2019;18(1):20.

53. Ba L, Xue C, Li X, et al. Gastric cancer cell-derived exosomes can regulate the biological functions of mesenchymal stem cells by inducing the expression of circular RNA circ_0004303. Stem Cells Dev 2021;30(16):830-842.

54. Wang Y, Wang H, Zheng R, et al. Circular RNA ITCH suppresses metastasis of gastric cancer via regulating miR-199a-5p/Klotho axis. Cell Cycle. 2021;20(5-6):522-536.

55. Jiang J, Li R, Wang J, Hou J, Qian H, Xu W. Circular RNA cdr1as inhibits the metastasis of gastric cancer through targeting miR-8765p/GNG7 axis. Gastroenterol Res Pract. 2021;2021:5583029.

56. Exosome profiling pinpoints cancer type. Cancer Discov. 2020;10 (11):1619. doi: $10.1158 / 2159-8290$
57. Shao Y, Tao X, Lu R, et al. Hsa circ 0065149 is an indicator for early gastric cancer screening and prognosis prediction. Pathol Oncol Res. 2020;26(3):1475-1482.

58. Tao X, Shao Y, Lu R, et al. Clinical significance of hsa circ 0000419 in gastric cancer screening and prognosis estimation. Pathol Res Pract. 2020;216(1):152763.

59. Yao W, Guo P, Mu Q, Wang Y. Exosome-derived circ-PVT1 contributes to cisplatin resistance by regulating autophagy, invasion, and apoptosis via miR-30a-5p/YAP1 axis in gastric cancer cells. Cancer Biother Radiopharm. 2021;36(4):347-359.

60. Liu S, Wu M, Peng M. Circ_0000260 regulates the development and deterioration of gastric adenocarcinoma with cisplatin resistance by upregulating MMP11 via targeting MiR-129-5p. Cancer Manag Res. 2020;12:10505-10519.

\section{Publish your work in this journal}

Cancer Management and Research is an international, peer-reviewed open access journal focusing on cancer research and the optimal use of preventative and integrated treatment interventions to achieve improved outcomes, enhanced survival and quality of life for the cancer patient.
The manuscript management system is completely online and includes a very quick and fair peer-review system, which is all easy to use. Visit http://www.dovepress.com/testimonials.php to read real quotes from published authors. 